\title{
Extreme cooperative swelling in topologically disordered fibre entanglements
}

Alistair Overy $^{1,3}$, Raj Pandya ${ }^{2}$, Phillip Maffettone ${ }^{1}$, Arkadiy Simonov ${ }^{1}$, Andrew Goodwin ${ }^{1}$

${ }^{1}$ Chemistry, University Of Oxford, Oxford, United Kingdom, ${ }^{2}$ Optoelectronics, University of Cambridge, Cambridge, United Kingdom,

${ }^{3}$ Diamond Light Source, Harrell, United Kingdom

E-mail: alistair.overy@chem.ox.ac.uk

Entangled states are ubiquitous amongst fibrous materials, whether naturally occurring (keratin, collagen, DNA) or synthetic (nanotube assemblies, elastane). A key mechanical response of these systems is their ability to dilate in response to external stimuli, as implicated in e.g. hydration-induced swelling of keratin fibrils in human skin [1]. Swelling in fibrous systems is understood in the context of ordered entanglements ('weavings'), where it is known to be a collective phenomenon allowed only for a handful of specific topologies [2]. Given this sensitivity to topology, it has always been puzzling how disordered entanglements-including those found in real materials-manage also to support cooperative dilation mechanisms. We use a combination of geometric and lattice-dynamical modelling to study the effect of disorder on swelling behaviour. The model system we devise spans a continuum of quantifiable topological defect concentrations and is bounded by ordered states whose swelling behaviour is already known to be either vanishingly small or extreme. We find antagonism between topological disorder and swelling behaviour only in the limit of ordered weavings. Instead the capacity for swelling surprisingly returns as defect concentration is maximised. Crucially, the extreme swelling response previously observed only for certain key weavings can be matched by that of disordered entanglements. We establish a firm link between topological disorder and mechanical flexibility that has implications not only for polymer physics but also for the broader understanding of collective phenomena in disordered systems.

[1] Norlén, L. and Al-Amoudi, A. (2004). J. Invest. Dermatol., 123, 715-732.

[2] Evans, M. E. and Hyde, S. T. (2011). J. R. Soc. Interface, 8, 1274-1280.

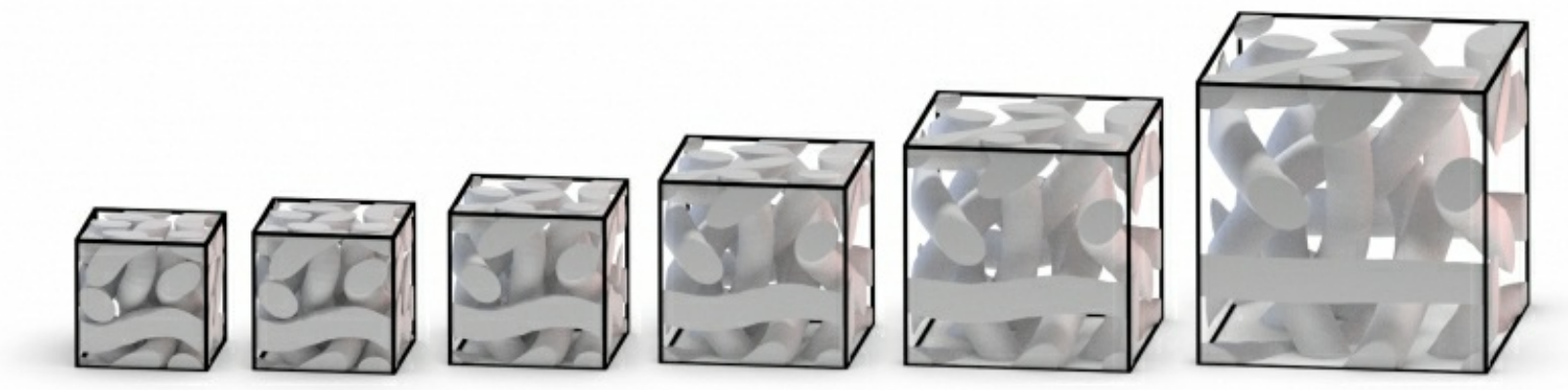

Keywords: rod packings, disorder, topology 